\title{
Avaliação do Efeito do Laser Preventivo na Mucosite Oral Quimioinduzida em Pacientes Submetidos a Altas Doses de Metotrexato
}

doi: https://doi.org/10.32635/2176-9745.RBC.2021v67n1.1128

\author{
Evaluation of the Effect of Preventive Laser on Chemo-induced Oral Mucositis in Patients Submitted to High Doses of \\ Methotrexate \\ Evaluación del Efecto del Láser Preventivo em la Mucositis Oral Inducida por Quimioterapia en Pacientes Sometidos a \\ Altas Dosis de Metotrexato
}

Lilian de Jesus Neves'; Érica Boldrini²; Hélio Massaiochi Tanimoto ${ }^{3}$; Deny Munari Trevisani ${ }^{4}$; Luiz Fernando Lopes ${ }^{5}$; Karina Silva Moreira Macari'

\begin{abstract}
RESUMO
Introdução: A quimioterapia, uma das formas de tratamento de neoplasias malignas, tem sua administração associada a inúmeras drogas, sendo uma delas o metotrexato (MTX), de alta toxicidade, responsável por inúmeros fatores agravantes para a saúde e bem-estar do paciente. Uma das principais complicaçóes é a mucosite oral, manifestaçáo clínica resultante do tratamento oncológico que pode interferir no tratamento e na cura. Objetivo: Avaliar, comparativamente, por meio de um estudo retrospectivo, o efeito do laser preventivo na ocorrência da mucosite oral quimioinduzida em pacientes com osteossarcoma não metastático submetidos a altas doses de MTX, bem como a intensidade da mucosite oral, utilizando o laser preventivo após os ciclos quimioterápicos contendo o medicamento MTX nos pacientes atendidos no Hospital de Câncer infantojuvenil de Barretos/SP. Método: Estudo de coorte com coleta retrospectiva em prontuários. Os pacientes foram divididos em dois grupos, um submetido à terapia profilática com laser de baixa intensidade após infusão do MTX e outro grupo não submetido a essa terapia. Resultados: Os dados obtidos mostraram que houve redução da gravidade da mucosite oral com o uso da laserterapia preventiva, com resultados estatisticamente significativos ( $<<0,001)$, corroborando os resultados encontrados na literatura. Conclusáo: $\mathrm{O}$ uso da laserterapia é uma terapêutica auxiliar importante na prevenção e na redução da severidade da mucosite oral em pacientes submetidos a altas doses de MTX, diminuindo o número de internaçóes por mucosite e os atrasos no protocolo terapêutico, o que reduz gastos e melhora o prognóstico para o paciente.
\end{abstract}

Palavras-chave: Estomatite/tratamento farmacológico; Metotrexato; Terapia com Luz de Baixa Intensidade; Odontopediatria.

\section{ABSTRACT}

Introduction: Chemotherapy, one of the treatments for malignant neoplasms, is associated to innumerous drugs, one of them methotrexate (MTX), of high toxicity, responsible for several health damages and impact on the patient's well-being. One of the main complications is oral mucositis, a clinical manifestation resulting from the oncologic treatment that can interfere in the treatment and cure. Objective: To evaluate comparatively through a retrospective study, the effect of preventive laser in the occurrence of chemo-induced oral mucositis in patients with non-metastatic osteosarcoma submitted to high doses of methotrexate (MTX), and the intensity of oral mucositis, using the preventive laser after the chemotherapy cycles containing the drug methotrexate (MTX) in the patients treated at the Child and Adolescent Cancer Hospital of Barretos/SP. Method: Retrospective cohort study with charts review. The patients were divided in two groups, one submitted to low-intensity laser prophylaxis therapy after infusion of MTX and another group not submitted to prophylactic therapy. Results: The data obtained showed that preventive laser-therapy reduced the severity of oral mucositis with statistically significant results $(\mathrm{p}<0.001)$, corroborating the results found in the literature. Conclusion: The use of laser therapy is an important auxiliary therapy in the prevention and reduction of severity of oral mucositis in patients submitted to high doses of MTX, reducing the number of hospitalizations and delays in therapeutic protocol, which reduces costs and improves the patient prognosis.

Key words: Stomatitis/drug therapy; Methotrexate; Low-Level Light Therapy; Pediatric Dentistry.

\section{RESUMEN}

Introducción: La quimioterapia, es uma de las formas de tratamiento de las neoplasias malignas, tiene su administración asociada a numerosas drogas siendo una de ellas el metotrexato (MTX), de alta toxicidad, responsable de numerosos factores agravantes para la salud y bienestar del paciente. Una de las principales complicaciones es la mucositis oral, manifestación clínica resultante del tratamiento oncológico que puede interferir en el tratamiento y cura. Objetivo: Evaluar, comparativamente, a través de um estudio retrospectivo, el efecto del láser preventivo em la aparición de la mucositis oral quimio inducida em pacientes com osteosarcoma no mestastásico sometido a altas dosis de MTX, bien como la intensidade de la mucositis oral, utilizando el láser preventivo después de los ciclos quimioterápicos que contiene el medicamento MTX en los pacientes antendidos en el Hospital del Cáncer Infantojuvenil de Barretos/SP. Método: Estudio de coorte con colección retrospectiva en prontuários. Los pacientes fueron divididos em dos grupos, uno sometido a terapia profiláctica con láser de baja intensidade después de la infusión de MTX y otro grupo no sometido a terapia profiláctica. Resultados: Los dados obtenidos mostraron que hubo una reducción en la severidad de la mucositis oral con el uso de la terapia láser preventiva, con resultados estáticamente significativos $(\mathrm{p}<0,001)$, corroborando los resultados encontrados em la literatura. Conclusión: El uso de la terapia con láser es una terapia auxiliar importante en la prevención y reducción de la severidad de la mucositis oral em pacientes sometidos a altas dosis de MTX, diminuendo el número de internaciones por mucositis y retrasos en el protocolo terapéutico, lo que reduce los gastos y mejora el pronóstico para el paciente.

Palabras clave: Estomatitis/tratamiento farmacológico; Metotrexato; Terapia por Luz de Baja Intensidad; Odontología Pediátrica.

${ }^{1-6}$ Hospital de Câncer Infantojuvenil de Barretos. Departamento de Odontologia. Barretos (SP), Brasil.

1E-mail: lilijneves@hotmail.com. Orcid iD: https://orcid.org/0000-0001-5194-4368

2E-mail: boldrinierica@gmail.com. Orcid iD: https://orcid.org/0000-0001-8450-8730

3E-mail: heliotanimoto@uol.com.br. Orcid iD: https://orcid.org/0000-0003-1862-5466

${ }^{4} \mathrm{E}$-mail: denymt@hotmail.com. Orcid iD: https://orcid.org/0000-0002-2104-952X

${ }^{5}$ E-mail: If.lopes@yahoo.com. Orcid iD: https://orcid.org/0000-0001-9737-9245

${ }^{6}$ E-mail: karinamacari@hotmail.com. Orcid iD: https://orcid.org/0000-0002-7220-0074

Endereço para correspondência: Lilian de Jesus Neves. Rua Antenor Duarte Vilela, 1120 - Paulo Prata. Barretos (SP), Brasil. CEP 14784-370. E-mail: lilijneves@hotmail.com 


\section{INTRODUÇÃO}

Cerca de $40 \%$ dos pacientes que fazem quimioterapia desenvolvem efeitos colaterais bucais e esse número eleva-se para mais de $90 \%$ quando a criança se encontra abaixo dos 12 anos de idade ${ }^{1}$. Várias complicaçôes bucais podem ser observadas durante o tratamento oncológico, como a mucosite, a xerostomia, a disgeusia, a cárie de radiação e a osteorradionecrose ${ }^{2}$.

A principal alteração na cavidade bucal, resultante do tratamento oncológico, é a mucosite oral, que é definida como inflamação da mucosa bucal, podendo ser induzida tanto pela quimioterapia ${ }^{3}$ quanto pela radioterapia. Pode iniciar com o ressecamento bucal evoluindo para eritema e ulceraçôes, causando dor e dificuldade de deglutição, e envolver todo o trato gastrointestinal, além de favorecer a presença de infecçôes oportunistas ${ }^{4,2}$. A mucosite ocorre em virtude da ação dos fármacos e da radioterapia na proliferação celular, maturação e substituição. $\mathrm{O}$ efeito indireto possui associação com as drogas mielossupressoras, desregulando o sistema imunológico e processo de reparaçáo 5 .

O metotrexato (MTX) é um antimetabólito do ácido fólico e age sobre a fase $S$ da mitose. É um quimioterápico altamente tóxico para o organismo e muito relacionado ao aparecimento da mucosite, sendo utilizado em altas doses no tratamento de osteossarcoma ${ }^{6}$. Este é um tipo de tumor ósseo raro abaixo dos 5 anos de idade ${ }^{7}$, tendo seu pico na adolescência quando o crescimento é acentuado ${ }^{4,8,9}$.

O efeito colateral do tratamento antineoplásico, associado à mucosite, tende a causar uma série de fatores agravantes, ocasionando desconforto e dor, podendo haver limitação nutricional, levando à interrupção ou à modificação da administraçáo da medicação, aumentando o tempo de internação hospitalar, gerando mais custos e, em alguns casos, o risco de morte é considerável ${ }^{2,10-12}$.

A mucosite bucal pode ser classificada em quatro graus diversos, de acordo com o sistema de graduação da Organização Mundial da Saúde (OMS): grau 0 - indica ausência de alteraçóes; grau I - presença de eritema; grau II - presença de eritema, úlceras e alimentação sólida; grau III - úlceras e alimentação líquida; e grau IV - não consegue realizar alimentação via oral ${ }^{13}$.

Diversos são os tratamentos para a mucosite oral descritos na literatura, como a utilizaçáo da laserterapia de baixa intensidade, que apresenta efeito analgésico, anti-inflamatório e reparador ${ }^{14-17}$. A laserterapia tem sido utilizada tanto no tratamento quanto na prevenção da mucosite. Inúmeros trabalhos demonstram a diminuição da intensidade da mucosite oral quando o laser preventivo é utilizado, embora não haja um consenso quanto à potência e ao protocolo a ser utilizado ${ }^{1}$. Os protocolos fundamentados em evidências clínicas, desenvolvidos pela Associaçáo Multinacional de Cuidados de Suporte em Câncer (MASCC), recomendam que a terapia com a fotobiomodulação é relevante no decréscimo da dor e na reduçáo da incidência de mucosite oral em pacientes que foram submetidos a altas doses de quimioterapia para transplante de células-tronco hematopoiéticas e em pacientes submetidos à radioterapia em regiáo de cabeça e pescoço durante o momento de imunossupressão ${ }^{18-22}$.

A laserterapia tem ação em efeitos biológicos, como controle de dor e ação moduladora da inflamação, apresenta capacidade de modulaçáo de uma gama de eventos metabólicos celulares e tem propriedade curativa $^{11,12,20-22}$.

Os lasers de baixa intensidade aumentam o metabolismo celular, estimulando a atividade mitocondrial, atuando como analgésicos, anti-inflamatórios e reparadores da lesão na mucosa. Eles provocam diversos eventos biológicos, a exemplo da proliferaçáo epitelial e de fibroblastos, bem como sua maturação, locomoção e transformação em miofibroblastos. Há, também, alteraçôes celulares e vasculares que dependem, entre outros fatores, do comprimento de onda do laser. Ainda ocorre produção de colágeno, elastina e proteoglicanos, revascularização, contração da ferida, aumento da fagocitose pelos macrófagos, aumento da proliferação e ativação dos linfócitos e da força de tensão, acelerando o processo cicatricial ${ }^{14-16}$.

É relevante a importância do cirurgião-dentista na equipe multiprofissional de um hospital oncológico visando ao diagnóstico, à prevenção, ao manejo da mucosite oral e a demais complicaçóes bucais. Os cuidados odontológicos devem ser direcionados para promover e manter a integridade do tecido e prevenir complicaçóes bucais, melhorando a qualidade de vida, diminuindo o tempo de internaçáo hospitalar e os custos com o tratamento $^{11,16}$.

O presente trabalho teve por objetivo avaliar comparativamente, por meio de um estudo retrospectivo, o efeito do laser preventivo na ocorrência da mucosite oral quimioinduzida em pacientes com osteossarcoma não metastático submetidos a altas doses de MTX, atendidos no Hospital de Câncer Infantojuvenil de Barretos/SP.

\section{MÉTODO}

Estudo de coorte com coleta retrospectiva de prontuários, com populaçáo constituída por pacientes diagnosticados com osteossarcoma não metastático ao diagnóstico, submetidos ao protocolo GLATO (quimioterapia - doxorrubicina, cisplatina, dexametasona e MTX + cirurgia), atendidos no Hospital de Câncer 
Infantojuvenil de Barretos/SP, sendo acompanhados pelo setor odontológico. Os pacientes foram divididos em dois grupos: 1) pacientes tratados ou em tratamento para osteossarcoma não metastático que receberam laserterapia preventiva de três a cinco sessóes a partir da infusão do quimioterápico MTX a partir de 2012;2) pacientes tratados para osteossarcoma não metastático que não receberam laser preventivo durante o tratamento quimioterápico; ou seja, no período anterior ao ano de 2012.

O equipamento utilizado para realizar a laserprofilaxia foi o MM Optics Twin Laser, laser diodo, luz vermelha, com comprimento de onda de $660 \mathrm{~nm}, 100 \mathrm{mw}$ de potência, spot de saída de $0,03 \mathrm{~cm}^{2}$, no tempo de dez segundos, totalizando uma dose de energia de $33,3 \mathrm{~J} / \mathrm{cm}^{2}$.

Os pacientes receberam atendimento no Centro Infusional (D0) e no consultório odontológico (demais aplicaçôes), sendo utilizadas as normas de proteção oculares universais preconizadas para o uso do laser terapêutico. As aplicaçôes de laser foram realizadas logo após a infusão do MTX (D0) e durante três a cinco dias consecutivos, com intervalo de 24 horas. As áreas de aplicação pontual foram mucosas jugais e labiais, palato mole, assoalho bucal, bordo e ventre lingual, em contato direto, distribuídos em 36 pontos. A avaliaçáo da incidência e da gravidade da mucosite oral foi realizada com parâmetros da escala da OMS por dez dias consecutivos desde o início dos sintomas até o seu desaparecimento.

O estudo foi submetido e aprovado pelo Comitê de Ética em Pesquisa com Seres Humanos do Hospital de Câncer de Barretos/SP, sob o no ${ }^{\circ}$. CAAE: 64161817.5.0000.5437 e parecer $n^{\circ} .1 .905 .452$, com base na Portaria no ${ }^{\circ} .466 / 12$ do Conselho Nacional de Saúde e da Declaração de Helsinque.

O cálculo amostral foi desenvolvido baseado na média do escore de mucosite. De acordo com Cowen et al. ${ }^{23}$, o grupo de pacientes que náo recebeu laser preventivo teve média do escore de mucosite de 225,7 (desvio-padrão: 40,5), esperando uma diminuição de 50 pontos na média do escore de mucosite nos pacientes que receberem o laser preventivo, gerando entáo um effect size de 1,23. A comparação da média entre dois grupos independentes pelo teste T, com poder de $80 \%$, nível de significância de $5 \%$ e uma possível perda de informação de $10 \%$, resultou em um tamanho amostral de 12 participantes em cada grupo, totalizando 24 participantes no projeto.

Em funçáo do tamanho da amostra $(\mathrm{n}=24)$, testes não paramétricos foram empregados. O programa REDcap foi utilizado para geraçáo dos resultados apresentados ${ }^{24}$.

Os dados foram subordinados à análise estatística descritiva por intermédio da distribuição de frequências univariadas e bivariadas e das medidas descritivas médias e desvio-padrão. Para verificar associações entre as variáveis, utilizaram-se os testes qui-quadrado de Pearson e Exato de Fischer, considerando o nível de significância estatística de $5 \%$. As análises estatísticas foram realizadas com o uso do software SPSS v.21.0.

\section{RESULTADOS}

A amostra total foi composta por 24 indivíduos que realizaram o tratamento de osteossarcoma no Hospital de Câncer Infantojuvenil de Barretos nos anos de 2009 até 2017. Os pacientes que compuseram essa amostra foram divididos de forma sequencial em dois grupos, com 12 participantes cada, no grau de alocação de pacientes que foram submetidos à terapia com laser e pacientes que não se submeteram a essa terapia, totalizando 288 episódios de avaliações entre as semanas de infusão do MTX, em que cada paciente realizou 12 semanas do quimioterápico. Quanto à caracterização sociodemográfica da amostra, $58,3 \%$ dos participantes eram do gênero masculino e $41,7 \%$, do gênero feminino. Os pacientes, em sua maioria, eram procedentes da Regiấo Sudeste, principalmente do Estado de São Paulo (28,6\%).

Dos participantes que se autodeclararam brancos, oito eram do grupo que não foi submetido à terapia com laser e quatro do grupo submetido a tal terapia, sendo a autodeclaraçáo como parda inversamente proporcional, quatro no grupo sem a terapêutica do laser e oito no grupo submetido a essa mesma terapêutica. Em relação ao diagnóstico nutricional, o grupo sem laser apresentou $50,0 \%$ dos pacientes desnutridos ou com baixo peso, $33,3 \%$ com eutrofia e $16,7 \%$ com sobrepeso ou obeso. Já os participantes do grupo com laser foram 50,0\% desnutridos ou de baixo peso, $16,7 \%$ eutróficos e $33,3 \%$ com sobrepeso ou obeso, denotando um equilíbrio nutricional entre os grupos.

Comparando os graus de mucosite oral I e II entre os grupos, observa-se que os participantes do grupo com laser apresentaram maior número de mucosite (41), comparados aos participantes do grupo sem laser (10); já a mucosite oral grave (III e IV) foi observada em maior número no grupo que não foi submetido à terapia com laser (9). Nota-se também que sete episódios de mucosite não puderam ser graduados, conforme Tabela 1 .

$\mathrm{Na}$ Figura 1, verificam-se a distribuição dos episódios de mucosite oral e seus escores de graduação com grau de significância $(\mathrm{p}<0,001)$.

Quando comparados os pacientes do grupo com laser e do sem laser quanto à internaçâo por causa da mucosite, verificou-se que o grupo sem laser apresentou um aumento de 4,9\% em relação ao grupo de pacientes que fizeram o uso da terapia com o laser. Utilizando o teste estatístico qui-quadrado de Pearson, não foi encontrada relevância 
Tabela 1. Distribuição total de números e percentuais da graduação de mucosite oral entre os grupos

\begin{tabular}{lcccc}
\hline & \multicolumn{2}{c}{$\begin{array}{c}\text { Grupo sem } \\
\text { laser }\end{array}$} & \multicolumn{2}{c}{$\begin{array}{c}\text { Grupo com } \\
\text { laser }\end{array}$} \\
\hline Mucosite oral & $\mathbf{N}$ & $\%$ & $\mathbf{N}$ & $\%$ \\
\hline Ausente & 119 & $82,6 \%$ & 99 & $68,8 \%$ \\
Grau 1 & 2 & $1,4 \%$ & 23 & $16,0 \%$ \\
Grau 2 & 8 & $5,6 \%$ & 18 & $12,5 \%$ \\
Grau 3 & 5 & $3,5 \%$ & 1 & $0,7 \%$ \\
Grau 4 & 4 & $2,8 \%$ & 2 & $1,4 \%$ \\
Sem graduação & 6 & $4,2 \%$ & 1 & $0,7 \%$ \\
\hline
\end{tabular}

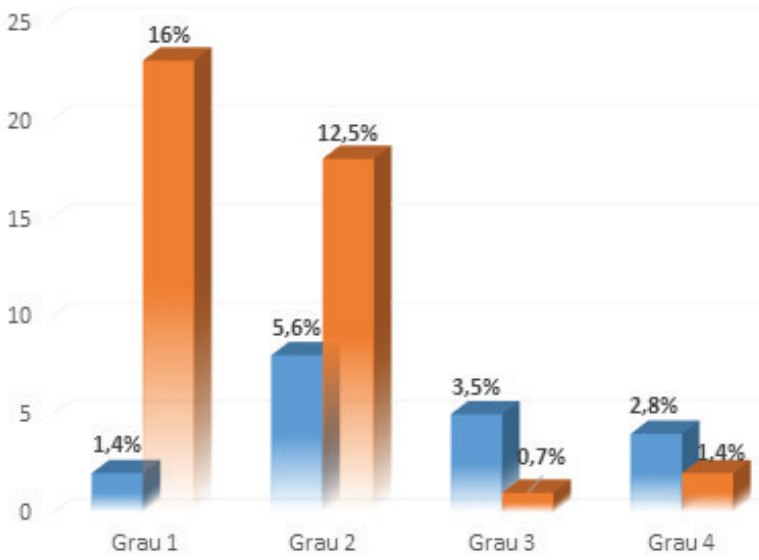

$=$ GRUPO SEM LASER $=$ GRUPO COM LASER

Figura 1. Episódios de mucosite oral em relação ao grau de intensidade, entre os grupos $(p<0,001)$ estatística quando comparados os dois grupos $(\mathrm{p}=0,06)$, como mostra a Tabela 2 .

$\mathrm{Na}$ avaliação do atraso do protocolo em razão da mucosite oral, os resultados mostraram que houve aumento do número de episódios de atraso no protocolo entre os pacientes que não fizeram o laser. Foram encontrados 11 episódios no grupo sem laser e um no grupo com laser. Utilizando o teste estatístico qui-quadrado de Pearson, foi encontrada diferença estatisticamente significativa quando comparados os dois grupos $(\mathrm{p}=0,005)$, conforme descrito na Tabela 3.

$\mathrm{Na}$ avaliação da presença de mucosite oral em relação às toxicidades apresentadas, os resultados mostraram que houve aumento do número de episódios de mucosite oral nos pacientes que tiveram alteraçôes hepáticas, com relevância estatística $(\mathrm{p}=0,001)$, não sendo observadas alteraçôes significativas em relação a náuseas, vômitos, neutropenia, disfunção renal e plaquetopenia, de acordo com a Tabela 4.

\section{DISCUSSÃO}

O presente estudo avaliou o efeito do laser preventivo na mucosite oral quimioinduzida em pacientes submetidos a altas doses de MTX, para tratamento de osteossarcoma no Hospital de Câncer Infantojuvenil de Barretos/SP, com o objetivo de avaliar a resposta da mucosite a essa terapia.

A mucosite bucal é considerada uma condição clínica de alta morbidade em pacientes submetidos a altas doses de MTX e merece atenção especial multiprofissional e interdisciplinar no diagnóstico, tratamento e reabilitação do paciente, para o alcance da melhoria da qualidade de vida.

Tabela 2. Distribuição das internações por mucosite oral, por episódio, entre os grupos

\begin{tabular}{cccccccc}
\hline \multirow{2}{*}{ Internação por mucosite } & \multicolumn{2}{c}{ Grupo sem laser } & \multicolumn{2}{c}{ Grupo com laser } & \multicolumn{3}{c}{ Total } \\
\cline { 2 - 7 } & $\mathbf{N}$ & $\%$ & $\mathbf{N}$ & $\%$ & $\mathbf{N}$ & $\%$ \\
\hline Não & 135 & 93,8 & 142 & 98,6 & 277 & 96,2 \\
Sim & 9 & 6,3 & 2 & 1,4 & 11 & 3,8 \\
Total & 144 & 100 & 144 & 100 & 288 & 100 \\
\hline
\end{tabular}

Legenda: Valor de $\mathrm{p}=0,06$

Tabela 3. Distribuição do atraso no protocolo em razão da mucosite oral, por episódio, entre os grupos

\begin{tabular}{ccccccc}
\hline \multirow{2}{*}{ Atraso do protocolo } & \multicolumn{2}{c}{ Grupo sem laser } & \multicolumn{2}{c}{ Grupo com laser } & \multicolumn{2}{c}{ Total } \\
\cline { 2 - 7 } & $\mathbf{N}$ & $\%$ & $\mathbf{N}$ & $\%$ & $\mathbf{N}$ & $\%$ \\
\hline Não & 130 & 92,2 & 139 & 99,3 & 269 & 95,7 \\
Sim & 11 & 7,8 & 1 & 0,7 & 12 & 4,3 \\
Total & 141 & 100 & 140 & 100 & 281 & 100 \\
\hline
\end{tabular}

Legenda: Valor de $\mathrm{p}=0,005$ (estatisticamente significativo). 
Tabela 4. Distribuição da mucosite oral, em razão de fatores relacionados à toxicidade, entre os grupos

\begin{tabular}{cccccc}
\hline \multirow{2}{*}{ Toxicidades } & & \multicolumn{4}{c}{ Mucosite oral } \\
\cline { 3 - 6 } & & & Não & Sim \\
\cline { 3 - 6 } & & N & $\%$ & N & $\%$ \\
\hline \multirow{2}{*}{ Náusea } & Ausente & 35 & $100,0 \%$ & 219 & $86,9 \%$ \\
& Presente & 0 & $0,0 \%$ & 33 & $13,1 \%$ \\
Vômito & Ausente & 35 & $100,0 \%$ & 204 & $81,3 \%$ \\
& Presente & 0 & $0,0 \%$ & 47 & $18,7 \%$ \\
Neutropenia & Ausente & 15 & $41,7 \%$ & 137 & $54,4 \%$ \\
\multirow{2}{*}{ Disfunção hepática } & Presente & 21 & $58,3 \%$ & 115 & $45,6 \%$ \\
(p=0,001) & Ausente & 0 & $0,0 \%$ & 62 & $24,7 \%$ \\
& Presente & 36 & $100,0 \%$ & 189 & $75,3 \%$ \\
Disfunção renal & Ausente & 29 & $80,6 \%$ & 230 & $91,3 \%$ \\
& Presente & 7 & $19,4 \%$ & 22 & $8,7 \%$ \\
Plaquetopenia & Ausente & 29 & $80,6 \%$ & 222 & $88,1 \%$ \\
& Presente & 7 & $19,4 \%$ & 30 & $11,9 \%$ \\
\hline
\end{tabular}

Desse modo, a laserterapia configura-se como uma tecnologia de relevância no cuidado ao paciente oncológico, em especial aquele em idade pediátrica, melhorando a qualidade de vida, enquanto reduz o principal efeito adverso da terapêutica antineoplásica. A ênfase na equipe multiprofissional releva que a mucosite oral náo se restringe a uma única área de atuação profissional, havendo necessidade da integração de membros das várias partes que formam o corpo da Saúde, entre as quais, destaca-se a atuação da equipe de odontologia, visto que ela está em maior contato com a cavidade bucal do paciente.

Quanto ao perfil da população do estudo, $58,3 \%$ dos indivíduos eram do gênero masculino e $41,7 \%$ do feminino. Em sua maioria, eram procedentes da Região Sudeste, principalmente do Estado de São Paulo (28,6\%). Em relação à raça e ao diagnóstico nutricional, notou-se um equilíbrio entre os grupos.

A maioria dos pacientes $(75,69 \%)$ apresentou mucosite bucal em algum grau segundo a OMS. No presente estudo, houve prevalência de mucosite graus I e II nos pacientes submetidos à laserterapia profilática quando comparados aos pacientes que não se submeteram, isto se justifica em razáo do tipo de coleta retrospectiva do estudo. No grupo não submetido à terapia, alguns dados foram subestimados, principalmente em virtude da falta do acompanhamento odontológico anterior ao ano 2012, o qual só passou a ser mais efetivo após a implantação da equipe odontológica exclusiva no Hospital Infantojuvenil.

A mucosite grau II foi a mais observada, o que demonstra que a laserterapia de baixa potência, quando aplicada em pacientes submetidos a altas doses de MTX, é eficaz no controle da severidade da mucosite oral. Segundo a literatura, a mucosite é esperada em $40 \%$ a $80 \%$ dos pacientes em virtude da quimioterapia, sendo notório que as complicaçóes em cavidade bucal aumentam de $40 \%$ para $90 \%$ em crianças menores de 12 anos, por conta da divisão celular ser mais rápida ${ }^{1,2,7,9,20-22}$.

A laserprofilaxia e a laserterapia têm sido cada vez mais utilizadas nesse grupo de pacientes para prevenção e tratamento da mucosite bucal ${ }^{22,23}$. Inúmeros trabalhos demonstram preocupaçáo com a adequada utilizaçáo dessa modalidade terapêutica quanto ao tipo de laser, dose, frequência e momento de suas aplicaçốes $1,8,21,22$.

Observou-se, nesta pesquisa, por meio dos resultados obtidos, que houve clinicamente reduçáo da gravidade da mucosite oral, com resultados estatisticamente significativos $(\mathrm{p}<0,001)$, corroborando os resultados encontrados na literatura ${ }^{15,17,21,23,25}$.

É válido salientar que as medidas de cuidados bucais prévios à terapia quimioterápica são fundamentais para a redução dos sintomas nesses pacientes ${ }^{11}$. Neste estudo, não foi possível observar dados referentes aos cuidados de higiene bucal em virtude de a coleta ser retrospectiva e estes não serem devidamente registrados nos prontuários analisados.

Neste estudo, a maioria dos pacientes que apresentaram mucosite oral grave (graus III e IV), durante a infusão do MTX, não fez o laser preventivo. Esses resultados vão de encontro com a literatura, que mostra que pacientes submetidos à laserterapia profilática apresentam redução do grau de severidade da mucosite ${ }^{14,26,27}$.

Pacientes com mucosite oral grave internaram mais do que aqueles com mucosite graus I e II, indo de encontro 
com a literatura. Segundo Lopes et al. ${ }^{28}$, nos pacientes com mucosite bucal grave, o período de internação seria de dois a seis dias, mais longo do que entre aqueles pacientes com mucosite graus I e II ou ausente.

A mucosite oral também pode resultar em aumento do tempo de permanência do paciente internado no hospital, necessidades de cuidados especiais, incluindo infusão intravenosa de barbitúricos, outros fármacos, e nutrição parenteral, o que juntos levam a um custo econômico mais elevado ${ }^{15,17,21-23}$.

A eficiência da laserterapia tem sido demonstrada há mais de 30 anos na França, com pacientes tratados com quimioterápicos, incluindo o 5-fluorouracil e o MTX, cujas frequência e gravidade da mucosite oral foram significativamente reduzidas pela terapia com laser, e a incidência de complicaçóes orais diminuiu de $43 \%$ para $6 \%{ }^{22}$. Com a laserterapia reduzindo as comorbidades, o atendimento para tratamento de câncer foi otimizado e houve redução das interrupçóes não planejadas, garantindo a continuidade da quimioterapia ${ }^{3,22}$. A laserterapia profilática reduz o risco global de mucosite oral e de outras medidas da gravidade da mucosite, incluindo a duração da mucosite severa em pacientes com câncer ${ }^{14,22}$. Notou-se, também, uma redução relevante na quantidade de internaçôes por mucosite nos pacientes que fizeram a terapia com laser, encontrando um percentual de 1,4\%, enquanto, no grupo sem laser, o percentual encontrado foi de $6,3 \%$. Embora estatisticamente não tenha sido encontrado significância $(\mathrm{p}=0,06)$, verificou-se um aumento do número de internaçóes entre os grupos. No presente estudo, no grupo sem laser, observou-se interrupção dos ciclos quimioterápicos por conta da presença de mucosite oral em $7,8 \%$ dos episódios, enquanto, no grupo com laser, ocorreu apenas com $0,7 \%$, comprovando resultados estatisticamente significativos ( $\mathrm{p}=0,005)$.

No que se refere ao tempo de aparecimento e à resoluçáo da mucosite oral, a literatura traz que, com o uso do laser, há uma relevante redução na gravidade e no número de dias do processo de cicatrizaçãa ${ }^{22}$. Entretanto, esse é um dado que não foi possível ser avaliado no estudo, por este ser retrospectivo e as informaçóes relevantes não estarem descritas nos prontuários.

Quanto à toxicidade, a literatura traz informaçóes pertinentes, porém são eventos considerados esperados com o uso do MTX, conforme demostrado na Tabela 4; no entanto, neste estudo, notou-se um aumento do número de mucosite nos pacientes que apresentaram alteração na função hepática, fato que nos deixa reflexivos quanto à necessidade de considerar como relevante a elevaçáo desses parâmetros de toxicidade. Em relação à influência dos níveis séricos do MTX, pode-se inferir que alteraçôes nos resultados das dosagens influenciam no aparecimento da mucosite, tendo em vista que a excreção retardada ou a intoxicação aumentam o tempo de ação do fármaco no organismo, contudo, esse dado, em relação à dosagem sérica, não foi avaliado nesta pesquisa por ser retrospectiva, e alguns dados necessários para obter essa informaçáo não foram descritos nos prontuários em análise, fato que sugere a necessidade de um futuro estudo $5,9,21,27$.

Ao analisar as variáveis náuseas e vômitos, todos participantes que apresentaram mucosite também apresentaram náuseas e vômitos, isto se explica em virtude da ação irritante e da redução do $\mathrm{pH}$ pós-liberação do suco gástrico em decorrência do vômito ${ }^{5,21,27}$.

A pesquisa poderia apresentar mais indicadores clínicos se houvesse a inclusão do início e a resolução da mucosite, todavia, essas desvantagens não reduzem a generalizaçấo dos resultados para a população estudada, mas fortalece a necessidade de se fazer um estudo prospectivo.

\section{CONCLUSÃO}

Verificou-se neste estudo que o uso da laserterapia se mostrou uma terapêutica auxiliar importante na prevenção e na reduçáo da severidade da mucosite oral grave em pacientes submetidos a altas doses de MTX.

A intensidade da mucosite oral foi observada em maior grau (III, IV) nos pacientes do grupo que não fizeram a terapia profilática com o laser (significância estatística), sendo também observado maior número de episódios de internação por mucosite e interrupção do tratamento em razão da mucosite nesse grupo.

\section{CONTRIBUIÇÕES}

Todos os autores contribuíram na concepção el ou desenho do trabalho; na aquisição, análise e/ou interpretação dos dados; na redação e/ou revisão crítica; e na aprovação final da versão publicada.

\section{DECLARAÇÃO DE CONFLITO DE INTERESSES}

Nada a declarar.

\section{FONTES DE FINANCIAMENTO}

Não há.

\section{REFERÊNCIAS}

1. Melo Júnior WA, Silva Júnior EF, Calista AA, et al. A laserterapia na prevenção e tratamento da mucosite oral em oncologia pediátrica. Rev Enferm UFPE on line. 2016;10(7):2404-11. doi: https://doi.org/10.5205/19818963-v10i7a11296p2404-2411-2016 
2. Ribeiro ILA, Valença AMG, Bonan PRF. Odontologia na oncologia pediátrica. 2. ed. João Pessoa: Ideia; 2018.

3. Curra M, Soares Junior LAV, Martins MD, et al. Chemotherapy protocols and incidence of oral mucositis. An integrative review. Einstein (São Paulo). 2018;16(1):eRW4007. doi: https://doi.org/10.1590/ s1679-45082018rw4007

4. Instituto Nacional de Câncer. Diagnóstico precoce do câncer na criança e no adolescente [Internet]. 2. ed. rev. ampl. Rio de Janeiro: INCA; 2011 [acesso 2020 abr 10]. Disponível em: https://www.inca.gov.br/sites/ufu.sti. inca.local/files//media/document//diagnostico-precocecrianca-adolescente-2011.pdf

5. Javed F, Utreja A, Correa FOB, et al. Oral health status in children with acute lymphoblastic leukemia. Crit Rev Oncol Hematol. 2012;83(3):303-9. doi: https://doi. org/10.1016/j.critrevonc.2011.11.003

6. Lobão DS, Oliveira BM, Massara MLA, et al. Condiçóes da cavidade bucal e acompanhamento odontológico de crianças com leucemia linfocítica aguda. Rev Med Minas Gerais. 2008;18(4 Supl 1):S25-S32.

7. Malagutti W, organizador. Oncologia pediátrica: uma abordagem multiprofissional. São Paulo: Martinari; 2011.

8. Fundato CT, Petrilli AS, Dias CG, et al. Itinerário terapêutico de adolescentes e adultos jovens com osteossarcoma. Rev Bras Cancerol. 2012;58(2):197208. doi: https://doi.org/10.32635/2176-9745. RBC.2012v58n2.620

9. Garrocho-Rangel JA, Herrera-Moncada M, MárquezPreciado R, et al. Oral mucositis in paediatric acute lymphoblastic leukemia patients receiving methotrexatebased chemotherapy: case series. Eur J Paediatr Dent. 2018;19(3):239-42. doi: https://doi.org/ 10.23804/ ejpd.2018.19.03.13

10. Volpato LER, Silva TC, Oliveira TM, et al. Mucosite bucal rádio e quimioinduzida. Braz J Otorhinolaryngol. 2007;73(4):562-68. doi: https://doi.org/10.1590/ S0034-72992007000400017

11. American Academy of Pediatric Dentistry. Dental management of pediatric patients receiving immunosuppressive therapy and/or radiation therapy. Pediatr Dent. 2018;40(6):392-400.

12. Ribeiro ILA, Melo ACR, Limão NP, et al. Oral mucositis in pediatric oncology patients: a nested case-control to a prospective cohort. Braz Dent J. 2020;31(1):78-88. doi: http://doi.org/10.1590/0103-6440201802881

13. World Health Organization. WHO Handbook for Reporting Results of Cancer Treatment. Geneva: WHO; 1979. (WHO offset publication; no. 48).

14. Oberoi S, Zamperlini-Netto G, Beyene J, et al. Effect of prophylactic low level laser therapy on oral mucositis: a systematic review and meta-analysis. PLoS One. 9(9):e107418. doi: http://doi.org/10.1371/journal. pone. 0107418
15. Figueiredo ALP, Lins L, Cattony AC, et al. Laser terapia no controle da mucosite oral: um estudo de metanálise. Rev Assoc Med Bras. 2013;59(5):467-74. doi: https:// doi.org/10.1016/j.ramb.2013.08.003

16. Sasada INV, Munerato MC, Gregianin LJ. Mucosite oral em crianças com câncer: revisão de literatura. RFO UPF. 2013;18(3):345-50. doi: https://doi.org/10.5335/ rfo.v18i3.3338

17. Pinheiro ALB, Brugnera Júnior A, Zanin FAA. Aplicação do laser na odontologia. Sáo Paulo: Santos; 2010.

18. Karu TI, Kolyakov SF. Exact action spectra for cellular responses relevant to phototherapy. Photomed Laser Surg. 2005;23(4):355-61. doi: https://doi.org/10.1089/ pho.2005.23.355

19. Lalla RV, Bowen J, Barasch A, et al. MASCC/ISOO clinical practice guidelines for the management of mucositis secondary to cancer therapy. Cancer. 2014;120(10):1453-61. doi: https://doi.org/10.1002/ cncr. 28592

20. Eilers J, Million R. Prevention and management of oral mucositis in patients with cancer. Semin Oncol Nurs. 2007;23(3):201-12. doi: https://doi.org/10.1016/j. soncn.2007.05.005

21. Cunha CB. Avaliação da eficácia do tratamento para mucosite oral induzida por cinco-fluoracil, com uso de laser de baixa potência em diferentes comprimentos de onda [dissertação]. São Paulo: Universidade de São Paulo, Faculdade de Odontologia; 2010.

22. Sung L, Robinson P, Treister N, et al. Guideline for the prevention of oral and oropharyngeal mucositis in children receiving treatment for cancer or undergoing haematopoietic stem cell transplantation. BMJ Support Palliat Care. 2017;7(1):7-16. doi: https://doi. org/10.1136/bmjspcare-2014-000804

23. Cowen D, Tardieu C, Schubert M, et al. Low energy Helium-Neon laser in the prevention of oral mucositis in patients undergoing bone marrow transplant: results of a double blind randomized trial. Int J Radiat Oncol Biol Phys. 1997;38(4):697-703. doi: https://doi.org/10.1016/ s0360-3016(97)00076-x

24. Harris PA, Taylor R, Thielke R, et al. Research electronic data capture (REDCap)--a metadata-driven methodology and workflow process for providing translational research informatics support. J Biomed Inform. 2009;42(2):37781. doi: https://doi.org/10.1016/j.jbi.2008.08.010

25. Santos PSS, Coracin FL, Barros JCA, et al. Impact of oral care prior to HSCT on the severity and clinical outcomes of oral mucositis. Clin Transplant. 2011;25(2):325-8. doi: https://doi.org/10.1111/j.1399-0012.2010.01283.x

26. Monteiro JYM. Oncologia oral: prevenção e tratamento da mucosite [dissertação na Internet]. Porto: Universidade Fernando Pessoa; 2017 [acesso 2020 abr 10]. 89 p. Disponível em: https://bdigital.ufp.pt/ bitstream/10284/5981/3/PPG_22104.pdf 
27.Zadik Y, Arany PR, Fregnani ER, et al. Systematic review of photobiomodulation for the management of oral mucositis in cancer patients and clinical practice guidelines. Support Care Cancer. 2019;27(10):396983. doi: https://doi.org/10.1007/s00520-019-04890-2

28. Lopes NNF, Plapler H, Chavantes MC, et al. Cyclooxygenase- 2 and vascular endothelial growth factor expression in 5-fluorouracil-induced oral mucositis in hamsters: evaluation of two low-intensity laser protocols. Support Care Cancer. 2009;17(11):1409-15. doi: https:// doi.org/10.1007/s00520-009-0603-9

Recebido em 30/6/2020

Aprovado em 25/8/2020 